\title{
Ergebnisse neuerer Untersuchungen zur Würmlöß-Gliederung in Hessen
}

\author{
Von E. Schönhals, H. Rohdenburg und A. Semmel \\ Mit 1 Abbildung im Text
}

\begin{abstract}
Zusammenfassung: Die über dem letztinterglazialen Boden (Parabraunerde bzw. pseudovergleyte Parabraunerde) folgenden würmzeitlichen äolischen Sedimente können durch kennzeichnende Bodenhorizonte in 3 Abschnitte (Alt-, Mittel- und Jungwürm) gegliedert werden. Charakteristisch für das Altwürm sind Humuszonen, für das Mittelwürm neben einigen "Naßböden" braune Verlehmungszonen bis zu einer Mächtigkeit von $1,1 \mathrm{~m}$ und für das Jungwürm mehrere schwach ausgebildete, geringmächtige Verbraunungszonen und "Naßböden“. Den wichtigsten Leithorizont des Jungwürms bildet das Kärlicher Tuffbändchen, das in jüngster Zeit auch in Nordhessen aufgefunden wurde. Abschließend wird das in Hessen aufgestellte Gliederungsschema mit den Würmlöß-Gliederungen in anderen Teilen Europas verglichen und eine Parallelisierung versucht.

A bstract: The eolian sediments of the "Würm" following above the last interglacial soil Parabraunerde and pseudogleyed Parabraunerde respectively) can be divided - by characteristic soil horizons - into three sections (old, middle and young Würm). Typical for the old Würm are humic zones, for the middle Würm - besides several "Naßböden" browncoloured (weathered) zones of decalcification with a thickness of up to $1,1 \mathrm{~m}$, and for the young Würm several weakly developed thin browncoloured weathering zones and "Naßböden" (wet soils). The most significant key horizon of the young Würm is the Kärlich tuff layer which recently was also encountered in Northern Hessen. Finally, the division scheme estabilished in Hessen is compared with the Würmloess divisions in other countries of Europe.
\end{abstract}

\section{Einleitung}

Die Gliederung des Würmlösses in Hessen war bereits in der Vergangenheit Gegenstand quartärgeologischer Untersuchungen (E. SCHöNHALS 1945, 1950, 1951, 1959 und 1959a; H. Freising 1954). Von E. SchöNhals wurde erstmalig das Auftreten von Verbraunungshorizonten im Würmlöß erkannt und als stratigraphisches Gliederungskriterium verwendet (E. Schönhals 1950 u. 1951). Derselbe Autor weist außerdem bereits 1951 auf einen weiteren wichtigen Leithorizont im hessischen Würmlöß hin: das Kärlicher Tuffband. Nach den Untersuchungen von J. Frechen (1959) stammt diese Tufflage, die als solche schon W. Hess (1909) und F. MrChels (1931) bekannt war, von einem basaltischen „Ausbläser" im Neuwieder Becken. Der Kärlicher Tuff tritt in vielen Lößaufschlüssen Hessens und seiner Randgebiete auf (E. SCHöNHALS 1959). In jüngerer Zeit wurde er z. B. von B. Meyer und H. Rohdenburg im Gebiet südlich Kassel gefunden. Der Wert des Tuffes als stratigraphisches Hilfsmittel besteht vor allem darin, daß er erlaubt, nur schwach entwickelte "Verbraunungshorizonte" oder "Naßböden" im oberen Abschnitt der Lößprofile zu parallelisieren. Wenn trotzdem in der Vergangenheit bei der Würmlöß-Gliederung in Hessen voneinander abweichende Meinungen vertreten wurden (vgl. H. FREISING 1954), so war die Ursache dafür oft nicht eine irrtümliche Gleichsetzung stratigraphisch verschiedener Horizonte, sondern vielmehr eine unterschiedliche genetische Deutung gleicher Bildungen.

Die vorliegende Arbeit hält sich methodisch an den bereits von E. SchöNHALS und H. FreIsING im Untersuchungsraum beschrittenen Weg. In ihr werden Ergebnisse mitgeteilt, die in den letzten Jahren durch voneinander unabhängige Untersuchungen in zahlreichen, größtenteils neu entstandenen Lößaufschlüssen Nordhessens und des Rhein-MainGebietes gewonnen wurden. Die Verfasser beschränken sich dabei im allgemeinen auf die Angabe der im Gelände faßbaren Daten. Aus diesen wurde für jedes Arbeitsgebiet ein Sammelprofil konstruiert. Die ausführlichen Beschreibungen der Einzelprofile sowie die Mitteilung der Ergebnisse von Laboruntersuchungen bleibt späteren, getrennten Publikationen vorbehalten. 


\section{Zur Gliederung des Würmlösses im Rhein-Main-Gebiet (Abb. 1)}

1.1. Der an der Basis des Würmlösses liegende Boden der letzten Warmzeit zeichnet sich durch eine leuchtendbraune (Munselu feucht 7.5 - 10 YR 5/4) Farbe aus. Er besitzt ein polyedrisch-plattiges Gefüge und weist häufig Merkmale eines $\mathrm{B}_{\mathrm{t}}$-Horizontes auf. Sein Tongehalt erreicht stellenweise $40 \%$. Der Boden tritt in zwei Varianten (Subtypen) auf. In den heute relativ trockenen Gebieten der Rhein-Main-Niederung, in denen der Löß stark karbonathaltig ist, durchsetzen das Solum weißliche, senkrecht verlaufende adrige Leitbahnen, die mit $\mathrm{CaCO}_{3}$-Ausscheidungen gefüllt sind. An der Basis des Bodens ist ein deutlicher Lößkindelhorizont ausgebildet. In dem niederschlagsreicheren Raum des Taunusrandes, in dem der Karbonatgehalt des Lösses geringer ist, fehlen solche deutlichen $\mathrm{CaCO}_{3}$-Ausscheidungen. Hier ist der Boden von einer schwachen Marmorierung überprägt. Graue, senkrechte Leitbahnen mit orangefarbigen Säumen treten vor allem im oberen Profilbereich auf und sviegeln eine Beeinflussung durch Staunässe wider. Die Mächtigkeit des Solums erreicht in den trockenen Gebieten $1,10 \mathrm{~m}$, in den niederschlagsreicheren dringen horizontale Tonbänder bis $1,80 \mathrm{~m}$ tief in den Rißlöß ein. Bei diesen Angaben muß aber berücksichtigt werden, daß die Profile durchweg gekappt sind. Die ursprüngliche Mächtigkeit des BHorizontes war wahrscheinlich größer.

Für die stratigraphische Einstufung dieses Bodens ist entscheidend, daß er unter einer Lößdecke, die fast immer durch eine Anzahl schwächerer Bodenbildungen gut gegliedert werden kann, der erste Boden ist, der dem holozänen Boden an Intensität gleicht bzw. ihn sogar übertrifft.

Die nicht pseudovergleyte Variante des letztwarmzeitlichen Bodens wird als „Erbacher Boden“, die pseudovergleyte als „Homburger Boden“ bezeichnet.

1.2. An der Oberfläche des Erbacher Bodens ist häufig ein Horizont mit Karbonat- und schwarzen Sesquioxidkonkretionen ausgebildet. Über diesem Horizont liegt manchmal eine $0,20-0,50 \mathrm{~m}$ starke hellgraue, steinige und kalkhaltige Schlufflage mit vereinzelten Sesquioxidkonkretionen und Holzkohlebröckchen. Auch über dem Homburger Boden folgt meist eine graue Schlufflage. Diese scheint aber stärker gebleicht zu sein und in ihr liegen mehr und größere Sesquioxidkonkretionen als über dem Erbacher Boden. Es ist möglich, daß die graue Schlufflage nach ihrer Ablagerung durch Lessivierung aufgehellt wurde und als $A_{1}$ - bzw. $S A_{1}$-Horizont zu betrachten ist, der mit dem liegenden $\mathrm{B}_{+}-\mathrm{bzw}$. SB+-Horizont eine bodengenetische Einheit bildet. Die graue Schlufflage wird von einer dunkelgrauen, schluffigen Humuszone überdeckt, deren Mächtigkeit 0,8 m erreichen kann. Zwischen beiden ist eine Diskordanz ausgebildet; denn in einigen Aufschlüssen fehlt die Schluffschicht zwischen der Humuszone und dem letztwarmzeitlichen Boden, in anderen Lehmgruben durchschneidet die Humuszone den $\mathrm{B}_{+}-$Horizont und liegt unmittelbar auf Rißlöß bzw. auf anderen Sedimenten präwürmzeitlichen Alters. Für die Humuszone ist charakteristisch, daß sie eine lebhafte Braunfleckung aufweist, die vielleicht als Folge eines Humusabbaues gedeutet werden kann (H. Rohdenburg 1964). Die braunen Flecken haben konkavgezackte Ränder und unterscheiden sich deutlich von dem auch in diesem Bereich auftretenden Krotowinen, die mit Löß oder braunem Lehm gefüllt sind. Im Hangenden der (steinfreien!) Humuszone liegt entweder eine steinige, stark lößhaltige Fließerde oder steinfreier Löß, denen abermals eine braunfleckige, schluffige Humuszone folgt. Diese besitzt an ihrer Basis oft einen braunen Saum. Uber einer weiteren Fließerde oder Lößlage folgt dann schließlich in einigen Aufschlüssen ein dritter humoser Abschnitt, der bis 1,5 m mächtig wird und den im oberen Teil zahlreiche sandige Lagen durchziehen.

Der vorstehend beschriebene untere Teil des Sammelprofils ist zur Zeit am besten im Raume Wiesbaden-Hattersheim a. M. aufgeschlossen. In den Aufschlüssen außerhalb 


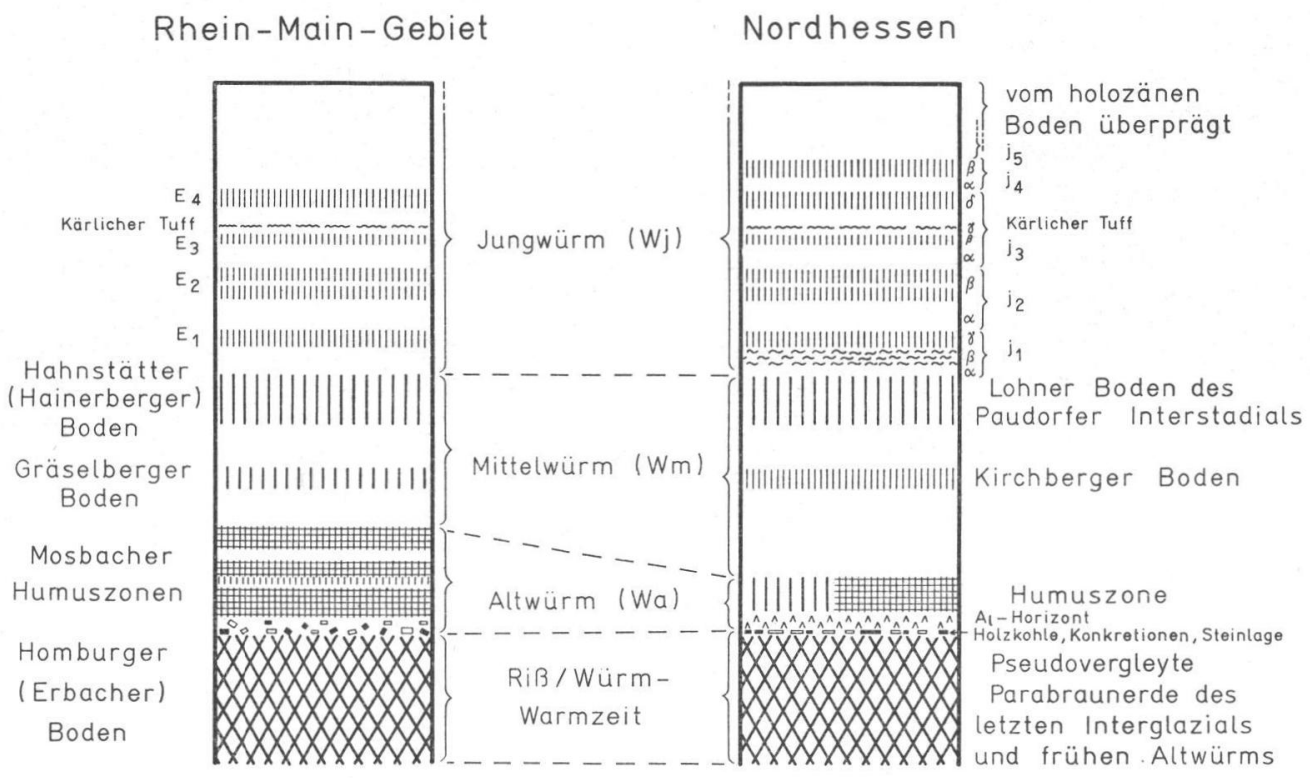

Abb. 1. Gliederung des Würm-Lösses im Rhein-Main-Gebiet und in Nordhessen.

dieses Gebietes fehlen entweder die Humuszonen völlig oder es ist nur eine von ihnen - wie z. B. in Hahnstätten (A. Semmel 1963) - ausgebildet bzw. erhalten geblieben. - Die Humuszonen werden als „Mosbacher Humuszonen“ bezeichnet.

1.3. Der über den Humuszonen folgende Löß ist in seinem basalen Teil durch einen braunen Lehm mit etwa 0,4 m Mächtigkeit gegliedert. Der Lehm besitzt ein plattig-bröckeliges Gefüge und Krotowinen, die mit hellem Löß gefüllt und von $\mathrm{CaCO}_{3}$-Pseudomyzelien durchsetzt sind. Der Tongehalt des Lehms beträgt $20-25 \%$, in seinem oberen Teil treten graue und rostfarbige Flecken auf. Wir bezeichnen diesen braunen Lehm als "Gräselberger Boden“. Das nach oben anschließende Lößpaket kann durch mehrere (meist vier) graue, etwas dichtere Horizonte, die sich gegenüber dem Löß durch einen geringfügig höheren Gehalt an Ton auszeichnen, gegliedert werden. Diese Lößserie wird durch einen etwa $0,80 \mathrm{~m}$ starken braunen Boden abgeschlossen, der in zwei Varianten auftritt. Dort, wo der Boden der letzten Warmzeit nicht pseudovergleyt ist, hat er eine schwach rötlich getönte Farbe (7.5 - 10 YR 5/4), dort, wo der letztwarmzeitliche Boden eine Marmorierung aufweist, entspricht die Farbe 10 YR 5/4. Im ersten Falle zeichnet sich der Boden durch ein plattig überprägtes Krümelgefüge aus, im zweiten Falle besitzt er ein plattig-polyedrisches Gefüge mit dunklen Anflügen auf den Aggregatflächen. In beiden Böden sind im oberen Profilteil häufig Krotowinen zu beobachten. Der Tongehalt beträgt 25-30\%. - Die rötliche Variante dieses Bodens wird als „Hainerberger Boden“, die bräunlichere als „Hahnstätter Boden" bezeichnet.

1.4. Der hierüber folgende Löß ist an der Basis grau- und rostfleckig. Er wird häufig durch vier schwache Verbraunungshorizonte unterteilt. Diese heben sich farblich nur wenig von dem übrigen Löß ab, sie besitzen aber ein dichteres Gefüge, sind stark mit Pseudomyzelien durchsetzt, manchmal schwach humos und etwas tonhaltiger. Durch ihren spezifischen Wasserhaushalt treten sie an der Aufschlußwand deutlich hervor. Der zweite Horizont (von unten gezählt) ist am kräftigsten und mächtigsten ausgebildet. Er weist oft eine deutliche Z $\mathrm{Z}$ weiteilung auf. An der Hangendgrenze des unteren Teiles 
ist stellenweise eine Quarzbröckchenlage zu erkennen. Der obere Teil zeichnet sich durch eine deutliche Grau- und Rostfleckung aus. Am schwächsten ist der dritte Horizont ausgebildet. Hier kann von einer Verbraunung nur sehr bedingt die Rede sein, denn häufig finden sich Beimengungen von lößfremdem Material (kleine Gesteinsbrocken), die den Horizont als Fließerde kennzeichnen. Der vierte Horizont ist fast immer von dem $\mathrm{C}_{\mathrm{a}}$-Horizont des holozänen Bodens überprägt und tritt nur undeutlich hervor. Zwischen dem dritten und vierten Horizont liegt das Kärlicher Tuffband. - Die schwachen „Verbraunungshorizonte“ werden als „Erbenheimer Böden“ bezeichnet (Symbole $\mathrm{E}_{1}-\mathrm{E}_{4}$ ).

1.5. Die vorstehend beschriebene Würmlöß-Serie ist zur Zeit nur am Osthang des Gräselberges südlich Wiesbaden aufgeschlossen. Im übrigen Untersuchungsgebiet scheint der basale Teil mit den Humuszonen nur sehr selten erhalten zu sein. Auch in dem darüber folgenden Würmlöß können Straten fehlen. In einigen Aufschlüssen schalten sich dagegen in Mulden zwischen zwei Verbraunungshorizonte mächtigere AbschwemmMassen ein.

\section{Zur Gliederung des Würmlösses in Nordhessen (Abb. 1)}

2.1. Der wichtigste Leithorizont ist ein Boden mit einem intensiv rötlichbraunen B-Horizont, der in der Regel eine Mächtigkeit von über 1,5 m erreicht, keinen Kalkgehalt aufweist und stark pseudovergleyt ist (vertikale Bleichbahnen mit orangefarbenen Säumen). Er besitzt eine polyedrische bis dichtplattige Struktur mit so hoher Strukturstabilität, daß die Fingerprobe einen viel zu niedrigen Tongehalt vortäuscht ${ }^{\top}$ ).

Darüber folgt stets ein mehrere Dezimeter mächtiger auffallend hellgrauer Horizont mit mehrere mm dicken, festen schwarzen Sesquioxidkonkretionen und kleinen Holzkohlestückchen.

Die Tonfraktion (unter $2 \mu$ ) entspricht zwar in ihrer Gesamtmenge etwa dem des normalen Lösses, besteht aber im Gegensatz zu diesem vorwiegend aus Grobton über $0,6 \mu$. Das deutet auf Tonbildung und nachfolgende korngrößenselektive Tonverlagerung hin. Dieser Horizont kann daher als $\mathrm{SA}_{1}$-Horizont („Bleichhorizont“) einer Parabraunerde (im weitesten Sinne) aufgefaßt werden.

Alle anderen Böden zwischen diesem Leithorizont und dem holozänen Boden sind in Farbintensität, Mächtigkeit usw. bedeutend schwächer ausgebildet. Der beschriebene B-Horizont wird demzufolge als letztinterglazial angesehen. Diese Einstufung findet darin eine Stütze, daß an der Oberkante des Bleichhorizontes Holzkohlen der Tanne (Abies) gefunden wurden, einer Baumart also, die in der Schlußphase von Interglazialen auftritt und die in der Nacheiszeit nicht bis Nordhessen vorgedrungen ist ${ }^{2}$ ). Es sprechen sehr viele Beobachtungen dafür, daß hier ein polygenetischer Boden vorliegt, in dem sich sowohl interglaziale wie auch frühkaltzeitliche Prozesse ausprägten. Zudem liegen in Steinchenlagen, „Hakenschlagen“ usw. genügend Kriterien vor, die eine aquatische und laminarsolifluidale Verlagerung zwischen einzelnen Teilphasen der Pedogenese beweisen.

2.2. Im Hangenden des Bleichhorizontes folgt in einigen Profilen eine humose Zone von wenigen Dezimetern bis zu über einem Meter Mächtigkeit. Dieser Profilabschnitt kann in Nordhessen nicht gegliedert werden; oft ist er nur sehr schwach ausgebildet oder er fehlt vollständig. In diesen Fällen tritt anstelle einer Humuszone ein meist mehrgliedriges Paket braunen Lehmes. Bei diesem handelt es sich aber nicht um eine Basisfließerde aus B-Horizont-Material des letztwarmzeitlichen Bodens, sondern um pedogenetisch stark überprägten und solifluidal bewegten Altwürmlöß.

1) Tongehalte bis $\mathrm{zu} 40 \%$.

2) Die Bestimmung der Holzkohlen verdanken wir Dr. U. Willerding, Göttingen. 
2.3. Darüber folgt ein Profilabschnitt, in dem eine vielfache Wechsellagerung von schwach bis mäßig braunem, etwas tonreicherem und „normalem“, hellgelbem Löß vorliegt. $\mathrm{Da}$ dieser Abschnitt sehr selten in größerer Mächtigkeit aufgeschlossen ist und die einzelnen braunen Schichten oder Bänder in der Regel etwas solifluidal verlagert sind, kann nichts über ihre Horizontbeständigkeit ausgesagt werden. Eine stratigraphische Bedeutung kommt womöglich der kräftigsten dieser braunen Zonen zu, die bei etwa $50 \mathrm{~cm}$ Mächtigkeit eine Farbe von 10 YR 5/6 besitzt und auf die ein „Naßboden“Horizont folgt. Wir bezeichnen diesen braunen Horizont als "Kirchberger Boden“.

Der zuletzt beschriebene Profilabschnitt wird von

2.4. einem stratigraphisch sehr wichtigen Leithorizont abgeschlossen. Es handelt sich um einen braunen Boden von durchschnittlich $70-100 \mathrm{~cm}$ Mächtigkeit. Er ist kalkfrei (bzw. sekundär wieder aufgekalkt) und besitzt einen wesentlich höheren Tongehalt als der primäre helle Löß und eine charakteristische plattige Struktur. Staunässeeinflüsse äußern sich in kennzeichnenden orangefarbenen weichen „Knötchenkonkretionen" und dünnen, schwärzlichen bis rötlichbraunen Sesquioxid-Belägen auf den Platten.

Dieser sogenannte „Lohner Boden“ ist wahrscheinlich nicht in seiner vollen Mächtigkeit von seiner heutigen Oberfläche her entstanden; eher ist anzunehmen, daß mehrere Phasen mit alternierender Materialzufuhr und Bodenbildung aufeinander folgten. Der untere etwa $40-50 \mathrm{~cm}$ mächtige Teil ist oft etwas farbkräftiger, außerdem tonund humusreicher als der obere Teil; mitunter zeigt er auch eine ausgeprägte plattige Struktur. Trotz dieses - nicht in jedem Profil zu beobachtenden - heterogenen Aufbaues hebt sich dieser Profilabschnitt als Einheit scharf vom Liegenden wie vom Hangenden ab. Infolge starker laminarsolifluidaler Beanspruchung verschuppt er sich aber mit beiden, an der Oberkante naturgemäß stärker als an der Unterseite.

In geringmächtigen Profilen treten in dem unter 2.3. beschriebenen Profilabschnitt keine hellen lockeren Lößlagen auf, sondern alles angewehte Material ist syn- oder paragenetisch verbraunt und entkalkt. Oft ist der durch den Lohner Boden abgeschlossene Abschnitt dann nur noch durch Plattigkeit und Knötchenkonkretionen von dem Liegenden zu trennen. Wenn die solifluidale Verlagerung zu stark war, ist keine Unterscheidung mehr möglich. Dann folgt ein mehr als $2 \mathrm{~m}$ mächtiges, entkalktes und verbrauntes, tonreicheres Bodenpaket iiber dem letztinterglazialen Boden.

2.5. Uber dem Lohner Boden wechseln mächtige Lagen aus hellem lockerem Löß mit schwach bis mäßig ausgeprägten Bodenhorizonten; es handelt sich dabei in der Regel um graubraune, kalkhaltige Zonen mit etwas erhöhtem 'Ton- und Humusgehalt, sowie geringerem Porenvolumen, stärkerer Frostblättrigkeit und schwacher Naßbodenüberprägung. Da diese Lößserie sehr häufig aufgeschlossen ist, konnte festgestellt werden, daß den einzelnen Horizonten stratigraphischer Wert zukommt. Sie besitzen nämlich z. T. charakteristische Kennzeichen. Der Horizont $J_{1} \gamma$ liegt stets über einem Lößpaket mit sehr starker Rostbänderung. Es ist noch fraglich, ob hier eine bodengenetische Horizontfolge vorliegt, oder ob sich in $\mathrm{J}_{1} \beta$ und $\mathrm{J}_{1} \gamma$ zwei getrennte Bodenbildungsphasen widerspiegeln. Die Zone $\mathrm{J}_{2} \beta$ ist sehr oft in zwei dünne graubraune Bodenzonen mit zwischengeschaltetem gelbem Löß zu gliedern und meistens recht schwach ausgebildet. Mitten in dem Lößpaket zwischen $\mathrm{J}_{2} \beta$ und $\mathrm{J}_{3} \delta$, das nur gelegentlich durch den schwachen Boden $\mathrm{J}_{3} \beta$ geteilt wird, liegt das Kärlicher Tuffband; in Nordhessen ist es günstigstenfalls nur noch $2 \mathrm{~mm}$ breit. Abgeschlossen wird dieses Lößpaket durch den in der Regel recht kräftig ausgebildeten Horizont $\mathrm{J}_{3} \delta$. Nur in wenigen Fällen ist noch der helle Löß $\mathrm{J}_{4} \alpha$ und der ebenfalls kräftige Bodenhorizont $\mathrm{J}_{4} \beta$ anzutreffen.

2.6. Der hangende Löß - in Ausnahmefällen $1 \mathrm{~m}$ mächtig - ist nirgends in seiner ursprünglichen Beschaffenheit erhalten, sondern überall von der holozänen Bodenbil- 
dung stark überprägt worden. Die Untergrenze der holozänen Entkalkung schwankt in ihrer Tiefenlage in Nordhessen sehr stark $(1,1$ bis über $3 \mathrm{~m})$. In sehr vielen Profilen ist $\mathrm{J}_{3} \delta$ schon entkalkt, so daß dann der Löß $\mathrm{J}_{3} \beta-\gamma$ als "C-Horizont" des holozänen Bodens angesprochen wird. Im unteren Teil des holozänen B-Horizontes heben sich dann $\mathrm{J}_{3} \delta$ und $\mathrm{J}_{4} \beta$ recht oft durch ihren deutlichen Grauton von dem reinen Braun der übrigen B-Horizontabschnitte ab. Bei größerer Entkalkungstiefe ist der B-Horizont der Parabraunerde nicht mehr völlig kompakt, sondern löst sich in viele getrennte Toninfiltrationsbänder auf, wie sie in Sand-Parabraunerden die Regel sind. Zwischen den Tonbändern treten infolge starker Tonverlagerung Bleichflecken auf, und zwar besonders stark im Bereich der schwachen pleistozänen Bodenhorizonte $\mathrm{J}_{3} \delta$ und $\mathrm{J}_{4} \beta$, sowie einiger Subhorizonte. Dadurch ergibt sich ein Profilbild, das von I. Lieberoth (1959) als „Lamellenfleckenzone“ beschrieben wurde. Diese hat aber nach Befunden, die an anderer Stelle ausführlich dargestellt werden sollen, in Nordhessen keinen stratigraphischen Wert, da ihre Ausbildung nicht syn-oder parasedimentär, sondern eindeutig postsedimentär erfolgte. Stratigraphisch wichtig sind nur die in ihr „entwickelten " fossilen Bodenhorizonte.

\section{Vergleich der Würmlöß-Gliederung zwischen dem Rhein-Main-Gebiet und Nordhessen}

In Abb. 1 sind die Sammelprofile beider Untersuchungsgebiete einander gegenübergestellt. Die in Nordhessen an der Basis des Würmlösses erhalten gebliebene Parabraunerde der letzten Warmzeit entspricht dem Homburger Boden des Rhein-Main-Gebietes. In beiden Gebieten ist über diesem Boden eine deutliche Diskordanz ausgebildet, die belegt, daß vor der Entstehung der hangenden grauen Schluffschicht die warmzeitliche Bodenbildung durch eine Abtragungsphase abgebrochen wurde. Da sich diese Abtragung auch auf nur schwach geneigten Flächen ausgewirkt hat, scheinen die abtragenden Vorgänge bereits kaltzeitlichen Charakter (Abspülung und Bodenfließen) besessen zu haben. Deshalb legen die Verfasser die Grenze Warmzeit/Altwürm an die Basis der grauen Schlufflage, obwohl in der Schlufflage später noch Lessivierung und im $\mathrm{B}_{\mathrm{t}}$-Horizont Toneinschlämmung und Marmorierung stattgefunden haben ${ }^{3}$ ).

Die Mosbacher Humuszonen fehlen in Nordhessen bis auf eine. Diese wird stellenweise durch einen braunen Lehm vertreten, der bisher im Rhein-Main-Gebiet in entsprechender stratigraphischer Position noch nicht beobachtet werden konnte.

Mit der dritten Humuszone schließt ein Sedimentationsabschnitt des Würmlösses ab, dessen spezifische Merkmale im darüber folgenden Löß nicht wiederkehren. Es scheint deshalb vertretbar, die Grenze Altwürm/Mittelwürm an die Oberkante der dritten Humuszone zu legen. Die Abgrenzung lehnt sich an den Vorschlag von P. Woldstedt (1962, S. 121) an.

Der „Kirchberger Boden“ Nordhessens scheint dem „Gräselberger Boden“ des RheinMain-Gebietes zu entsprechen. Die Parallelisierung von „Lohner Boden“ und „Hahnstätter Boden" kann als gesichert angesehen werden. Beide Böden gleichen sich in ihrer Ausbildung weitgehend. Sie repräsentieren die bedeutendste Wärmeschwankung innerhalb der letzten Kaltzeit; an ihre Oberkante legen wir die Grenze Mittelwürm/Jungwürm (vgl. P. WolDSTEDT 1962, S. 121). Auch die Jungwürm-Gliederung beider Gebiete entspricht sich weitgehend. Wenn trotzdem für die sich entsprechenden Horizonte verschiedene Bezeichnungen verwendet werden, so deshalb, weil gewisse Abweichungen nicht zu übersehen sind. Im

3) In der Ziegeleigrube von Witzenhausen/Werra konnte festgestellt werden, daß auch der interglaziale Boden vor der ersten kräftigen Abtragungsphase einen Bleichhorizont besaß und pseudovergleyt war. Darüber befindet sich auch dort ein durchgehender Bleichhorizont als altwürmzeitliche Neubildung. 
Rhein-Main-Gebiet ist z. B. der Horizont $\mathrm{E}_{1}$ sehr viel schwächer entwickelt als der Horizontkomplex E2. Dagegen zeichnen sich in Nordhessen die entsprechenden Horizonte ( $\mathrm{J}_{1} \gamma$ und $\mathrm{J}_{2} \beta$ ) durch etwa gleiche Intensität aus oder es ist $\mathrm{J}_{1} \gamma$ kräftiger entwickelt. Weiterhin ist der Horizont $\mathrm{J}_{3} \beta$ wesentlich schwächer ausgebildet als der Horizont $\mathrm{E}_{3}$. Ein dem $\mathrm{J}_{4} \beta$ äquivalenter Horizont konnte bisher im Rhein-Main-Gebiet nicht eindeutig beobachtet werden.

\section{Vergleich mit Würmlöß-Gliederungen in anderen Teilen Mitteleuropas}

Im Folgenden soll versucht werden, das Schema der Würmlöß-Gliederung in Hessen mit einigen in benachbarten Gebieten ausgearbeiteten Gliederungen zu vergleichen. Die Verfasser beschränken sich dabei auf Gliederungen, deren Standardprofile sie im Gelände selbst besichtigen konnten ${ }^{4}$ ).

Zunächst kann ohne Bedenken gefolgert werden, daß der „Homburger Boden“ dem „R/W-Boden“ K. Brunnacker's (u. a. 1958) und dem „Boden der Letzten Warmzeit“ im Sinne von H. Freising (u. a. 1957) stratigraphisch entspricht. Außerdem bestehen keine Zweifel darüber, daß der Homburger Boden mit den letztinterglazialen Bodenbildungen zu parallelisieren ist, wie sie von J. FinK (u. . 1962), B. KLIMA, J. KuKLA, V. LožEK \& H. de Vries (1962), I. Lieberoth (u. a. 1962), W. PaAs (1962), R. Ruske \& M. Wünsche 1961 und K. P. UNGER \& D. RAU (1959) beschrieben wurden (vgl. hierzu auch G. HAASE 1963).

Ganz ähnlich wie in bayerischen Lößprofilen (K. BrunNACKen 1958, S. 134-135) läßt sich auch in Hessen erkennen, daß das Bodenprofil der letzten Warmzeit bereits in einem frühen Stadium abtragenden Vorgängen ausgesetzt war. Im Gegensatz zu K. BRunNACKer, der diese Abtragungsphase einer feuchteren Periode des R/W-Interglazials zuordnet, neigen die Verfasser dazu, für diese Unterbrechung der typischen warmzeitlichen Bodenbildung Abspülung und Solifluktion verantwortlich zu machen, die ihre Ursache in einem Klima mit bereits kaltzeitlichem Charakter hatten. Nach Abschluß dieser Vorgänge setzte dann noch einmal eine Periode stärkerer Lessivierung und Pseudovergleyung ein. $\mathrm{Ob}$ während der vorausgegangenen Klimaverschlechterung schon Löß angeweht wurde, konnte bisher noch nicht geklärt werden. Dagegen entstand die erste Humuszone sicher zu einer Zeit, als bereits Löß akkumuliert wurde bzw. worden war. Diese Folgerung entspricht den Beobachtungen in der ČSSR und in Niederösterreich.

Die aus diesen Gebieten beschriebenen humosen Abschnitte des Altwürms lassen sich gut mit den Mosbacher Humuszonen parallelisieren. Hierzu ist um so mehr Berechtigung gegeben, als J. FINK (1962, S. 6) bemerkt, daß die mittlere Humuszone in Niederösterreich einen basalen braunen Saum besitzt. Diese Erscheinung konnte in einigen Aufschlüssen des Rhein-Main-Gebietes ebenfalls beobachtet werden. Sie ist auch in Niedersachsen, der ČSSR und in Thüringen bekannt. Wie schon erwähnt wurde, tritt in Nordhessen anstelle einer Humuszone manchmal ein brauner Lehm auf. Dieser ist dem braunen Lehm des „W $\alpha^{\text {“ }}$ von I. Lieberoth (1962, S. 189-190) gleichzusetzen.

Der „Kirchberger (Gräselberger) Boden“ besitzt in den außerhessischen Lößgebieten, soweit sie den Verfassern bekannt sind, kein sicheres Äquivalent. Es kann höchstens vermutet werden, daß er dem „FWc-Boden“ K. BRunNacken’s (u. a. 1958, S. 130) entspricht.

Von dem „Lohner (Hahnstätter) Boden“ ist dagegen mit Sicherheit anzunehmen, daß er dem „Braunen Verwitterungshorizont K. BrunnaCKen’s (u. a. 1959, S. 140), dem „Stillfried B“ bzw. „Paudorf“ J. FINK’s (u. a. 1961, S. 6-7), der "Gleinaer Bodenbildung“

4) Die Verfasser danken für freundliche Führung den Herren Prof. Dr. K. Brunnacker, Prof. Dr. J. Fink, Dr. H. Freising, Dr. G. Haase, Dr. I. Lieberoth, Dr. V. Ložek, Dr. W. Paas, Dr. D. Rau, Dr. K. P. Unger, Dr. M. Wünsche, Dr. K. Žebera. 
I. Lieberoth's (u. a. 1962, S. 190) und dem „PK I“ der tschechischen Lößforscher gleichzusetzen ist.

Die in Hessen im Jungwürmlöß auftretenden schwachen Bodenbildungen sind zur Zeit noch nicht mit denen der Nachbargebiete zu parallelisieren. Der Horizont „ $\mathrm{E}_{2}$ “ des RheinMain-Gebietes entspricht dem „Naßboden III" von H. Freising (u. a. 1957, S. 10). Dieser Boden ist z. B. in den Lößaufschlüssen Mühlacker und Böckingen gut ausgebildet und auf Grund seiner Lage zum Kärlicher Tuffband stratigraphisch ohne Schwierigkeiten zu fixieren.

\section{Literatur}

Brunnacker, K.: Zur Parallelisierung des Jungpleistozäns. - Geol. Jb. 76, S. 129-150, Hannover 1958.

FINK, J.: Die Gliederung des Jungpleistozäns in Ơsterreich. - Sonderdruck aus Mitt. geol. Ges. in Wien 54, Wien 1962.

Frechen, J.: Die basaltischen „Ausbläser" von Kärlich (Neuwieder Becken) und die Verbreitung ihrer Tuffe. - Fortschr. Geol. Rheinld. u. Westf. 4, S. 301-312, Krefeld 1959.

Freising, H.: Gibt es in Hessen drei Würmlösse? - Jber. u. Mitt. oberrh. geol. Ver., N. F. 35, S. 54-66, Stuttgart 1954. - - Neue Altsteinzzeitfunde aus Nordwürttemberg und ihr erdgeschichtliches Alter. - Fundber. aus Schwaben, N. F. 14, S. 7-27, Stuttgart 1957.

Hanse, G.: Stand und Probleme der Lößforschung in Europa. - Geogr. Ber. 27, S. 97-129, Berlin 1963.

Hess, W.: Uber die vulkanische Asche im Diluvium des Limburger Beckens. - Ber. Vers. niederrh. geol. Ver., Jg. 1909, S. 8-9, Bonn 1909.

Klima, B., J. Kukla, V. LožEK u. H. DE Vries: Stratigraphie des Pleistozäns und Alter des paläolithischen Rastplatzes in der Ziegelei von Dolni Věstonice (Unter-Wisternitz). - Anthropozoikum 11, S. 93-146, Praha 1962.

Lieberoth, I: Die jungpleistozänen Lösse Sachsens im Vergleich zu denen anderer Gebiete. - Pet. geogr. Mitt. 106, S. 188-198, Gotha 1962. - - Lößsedimentation und Bodenbildung während des Pleistozäns in Sachsen. - Geologie 12, S. 149-187, Berlin 1963.

Michels, F.: Erläuter. z. geol. Karte v. Preußen 1:25000, Blait Eltville-Heidenfahrt. - Bln. 1931.

PAAs, W.: Rezente und fossile Böden auf niederrheinischen Terrassen und deren Deckschichten. Eiszeitalter und Gegenwart 12, S. 165-230, O'hringen 1962.

Rohdenburg, H.: Ein Beitrag zur Deutung des „Gefleckten Horizontes“. - Eiszeitalter und Gegenwart 15, 6 S., Ohringen 1964.

Ruske, R. u. M. WÜNsche: Lösse und fossile Böden im mittleren Saale- und unteren Unstruttal. Geologie 10, S. 9-29, Berlin 1961.

SснӧNHALS, E.: Ubber verschiedenaltrige Lösse und ihre fossilen Verwitterungsdecken bei Homberg a. d. Efze (Bez. Kassel). - Ber. Reichsamt f. Bodenforsch. Jg. 1944, S. 111-119, Wien 1945. - - Uber einige wichtige Lößprofile und begrabene Böden im Rheingau. - Notizbl. hess. L.-Amt Bodenforsch. 6, S. 244-259, Wiesbaden 1950 - - Uber fossile Böden im nicht vereisten Gebiet. - Eiszeitalter und Gegenwart 1, S. 109-130, Öhringen 1951. - - Der Basalttuff von Kärlich als Leithorizont des Würm-Hochglazials. - Fortschr. Geol. Rheinld. u. Westf. 4, S. 313-322, Krefeld 1959. - - Klima, äolische Sedimentation und Bodenbildung während der Eiszeit in Hessen. - Z. deutsch. geol. Ges. 111, S. 251-25,3, Stuttgart 1959.

Semmel, A.: Mitteilung über ein Pleistozänprofil bei Hahnstätten (Limburger Becken). - Notizbl. hess. L.-Amt Bodenforsch. 91, S. 359-365, Wiesbaden 1963.

UnGER, K. P. u. D. RAU: Gliederung und Altersstellung der Lößablagerungen im Thüringer Becken und dessen Randgebiet. - Quaternary of Central and Eastern Europe, Teil I, S. 227-235, INQUA-Kongreß Warschau 1961.

Wornstedt, P.: Über die Gliederung des Quartärs und Pleistozäns. - Eiszeitalter und Gegenwart 13, S. $115-124$, Öhringen 1962.

Manuskr. eingeg. 4. 7. 1964.

Anschriften der Verf.: Prof. Dr. E. Schönhals, 3 Hannover, Wiesenstr. 1, Bundesanstalt für Bodenforschung. - - Dr. H. Rohdenburg, 63 Gießen, Geograph. Inst. d. Univ., Neues Schloß. - Dr. A. Semmel, 62 Wiesbaden, Leberberg 9-11, Hess. Landesamt für Bodenforschung. 